\title{
Midterm benefits of surgical pulmonary embolectomy for acute pulmonary embolus on right ventricular function
}

\author{
William Brent Keeling, MD, ${ }^{a}$ Bradley G. Leshnower, MD, ${ }^{a}$ Yi Lasajanak, MSPH, ${ }^{b}$ Jose Binongo, PhD, ${ }^{b}$ \\ Robert A. Guyton, MD, ${ }^{a}$ Michael E. Halkos, MD, ${ }^{a}$ Vinod H. Thourani, MD, ${ }^{a}$ and Omar M. Lattouf, MD $^{a}$
}

\section{ABSTRACT}

Objective: Surgical pulmonary embolectomy has been used for the successful treatment of massive and submassive pulmonary emboli. The purpose of this study is to document the short- and midterm echocardiographic follow-up of right ventricular function after surgical pulmonary embolectomy for acute pulmonary embolus.

Methods: A retrospective review of the local Society of Thoracic Surgeons database of patients who underwent surgical pulmonary embolectomy for acute pulmonary embolectomy was conducted from 1998 to 2014 at a US academic center. Patients with chronic thrombus were excluded. The institutional echocardiographic database was searched for follow-up studies to compare markers of right ventricular function. Unadjusted outcomes were described, and quantitative comparisons were made of short- and long-term echocardiographic data.

Results: A total of 44 patients were included for analysis; 35 patients $(79.5 \%)$ had a submassive pulmonary embolectomy, and 9 patients $(20.5 \%)$ had a massive pulmonary embolectomy and required preoperative inotropy. Mean cardiopulmonary bypass time was $68.0 \pm 40.2$ minutes, and 30 patients $(68.2 \%)$ underwent procedures without aortic crossclamping. There was 1 in-hospital mortality $(2.3 \%)$, and there were no permanent neurologic deficits. A total of 21 patients had echocardiography results available for follow-up. Perioperative echocardiographic data showed an immediate decrease in tricuspid regurgitant velocity and right ventricular pressure $(P<.05)$. Mean midterm echocardiographic follow-up was 30 months in 12 patients. At midterm follow-up, improvements in right ventricular function observed postoperatively persisted. Only 1 patient had moderate right ventricular dysfunction, and no patient had worse than mild tricuspid regurgitation. Mean tricuspid valve regurgitant velocity was $2.4 \pm 0.7 \mathrm{~m} / \mathrm{s}$, and mean pulmonary artery systolic pressure was $37.2 \pm 14.2 \mathrm{~mm} \mathrm{Hg}$.

Conclusions: Surgical pulmonary embolectomy may represent optimal therapy in selected patients for massive and submassive acute pulmonary emboli given the low morbidity and mortality rates. Echocardiographic follow-up shows preserved improvement in right ventricular function in the majority of patients. $(\mathrm{J}$ Thorac Cardiovasc Surg 2016;152:872-8)

\begin{tabular}{|c|c|c|c|}
\hline & $\begin{array}{c}\text { Preoperative Value } \\
(\mathrm{n}=\mathbf{2 1})\end{array}$ & $\begin{array}{c}\text { Mid-term Value } \\
(\mathrm{n}=\mathbf{2 1})\end{array}$ & P Value \\
\hline $\begin{array}{c}\geq \text { Moderate } \\
\text { ventricular } \\
\text { dysfunction }\end{array}$ & $17(81.0 \%)$ & $1(4.8 \%)$ & $<0.0001$ \\
\hline $\begin{array}{c}\geq \text { Moderate } \\
\text { tricuspid } \\
\text { regurgitation }\end{array}$ & $6(28.6 \%)$ & $0(0)$ & 0.0005 \\
\hline $\begin{array}{c}\text { Pulmonary Artery } \\
\text { Systolic Pressure } \\
\text { (mm/Hg) }\end{array}$ & $\begin{array}{c}51.2 \pm 21.6 \\
(\mathrm{n}=17)\end{array}$ & $\begin{array}{c}37.2 \pm 14.2 \\
(\mathrm{n}=9)\end{array}$ \\
\hline $\begin{array}{c}\text { Tricuspid Valve } \\
\text { Regurgitant } \\
\text { Velocity (m/s) }\end{array}$ & $\begin{array}{c}3.1 \pm 0.8 \\
(\mathrm{n}=16)\end{array}$ & $\begin{array}{c}2.4 \pm 0.7 \\
(\mathrm{n}=11)\end{array}$ & 0.01 \\
\hline
\end{tabular}

Midterm echocardiographic follow-up.

\section{Central Message}

SPE is safe, and the benefit to right ventricular function is durable over time.

\section{Perspective}

SPE for acute PE is not performed frequently enough in the treatment of life-threatening PE. These data show that pulmonary embolectomy is safe with low morbidity and mortality. Via midterm echocardiographic follow-up, they also demonstrate that the immediate benefits to right ventricular function persist over time.

See Article page 669.

See Editorial Commentaries page 675 and 879 .

See Editorial page 656.
From the ${ }^{\mathrm{a} C l i n i c a l}$ Research Unit, Division of Cardiothoracic Surgery, Joseph B. Whitehead Department of Surgery, and ${ }^{\mathrm{b}}$ Department of Biostatistics, Rollins School of Public Health, Emory University School of Medicine, Atlanta, Ga. This research was funded by the Emory University Department of Surgery.

Read at the 95th Annual Meeting of The American Association for Thoracic Surgery, Seattle, Washington, April 25-29, 2015.

Received for publication April 28, 2015; revisions received Oct 9, 2015; accepted for publication Nov 13, 2015; available ahead of print March 16, 2016.

Address for reprints: William Brent Keeling, MD, 49 Jesse Hill Drive, Atlanta, GA 30303 (E-mail: brent.keeling@emory.edu).

$0022-5223 / \$ 36.00$

Copyright (c) 2016 by The American Association for Thoracic Surgery

http://dx.doi.org/10.1016/j.jtcvs.2015.11.042
Pulmonary embolus (PE) remains a significant cause of mortality in the United States, with 60,000 to 200,000 mortalities per year as a result of a significant PE. ${ }^{1-4} \mathrm{PE}$ is the

Scanning this QR code will take you to the article title page. To view the AATS 2015 Webcast, see the URL at the end of the article.

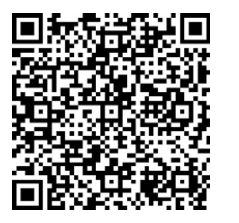




\section{Abbreviations and Acronyms \\ $\mathrm{PE}=$ pulmonary embolus \\ PERT $=$ pulmonary embolus response team \\ SPE = surgical pulmonary embolectomy}

third most common cause of cardiovascular mortality after myocardial infarction and stroke. ${ }^{5}$ An international registry with more than 2000 patients with pulmonary emboli detailed a 3-month mortality rate of $17.4 \%$ after an acute $\mathrm{PE}$, and half of those deaths were directly due to the PE. ${ }^{6}$ That same registry showed that of the more than 2000 cases of PE, only $108(4.5 \%)$ were massive PEs (patients who presented with a PE and a systolic blood pressure of $<90 \mathrm{~mm} \mathrm{Hg}$ ). ${ }^{7}$ Although the exact fate of right ventricular function is unclear after both massive and submassive pulmonary emboli, those patients with right ventricular dysfunction experience increased rates of mortality both during their hospital stay and at follow-up visits. ${ }^{8,9}$

Many therapies have been proposed for acute PE. The mainstay of initial treatment has been timely institution of systemic anticoagulation on discovery. ${ }^{10}$ However, there is little scientific agreement on the optimal therapy for submassive (systolic blood pressure $>90 \mathrm{~mm} \mathrm{Hg}$ but with evidence of right ventricular dysfunction or serologic cardiac injury) and massive pulmonary emboli beyond initial anticoagulation. Of the 108 patients with massive pulmonary emboli in the ICOPER registry, 33 underwent thrombolysis, 3 underwent surgical embolectomy, and 1 had catheterdirected embolectomy. The remaining patients received no treatment beyond systemic anticoagulation alone. ${ }^{7}$ There have been few randomized trials to address the issue of ideal treatment strategy for submassive and massive PEs, and none of them have included surgical pulmonary embolectomy (SPE) in the treatment arms.

SPE was first described by Trendelenburg but was ultimately moved forward with the advent of cardiopulmonary bypass as described by Sharp ${ }^{11}$ and Gibbon. ${ }^{12}$ Since that time, many single-center series have detailed indications and outcomes of SPE. ${ }^{13,14}$ Despite excellent outcomes on very ill patients, SPE as routine therapy has yet to be established, and midterm follow-up is lacking.

The purpose of this study was to review our institutional experience with SPE and detail current methods and results. Further, we sought to document the midterm echocardiographic follow-up of patients who underwent SPE to prove that the immediate postoperative improvements in right ventricular function were durable.

\section{MATERIALS AND METHODS \\ Study Population}

A retrospective review of the local Society of Thoracic Surgeons database of consecutive patients who underwent SPE for acute PE was conducted from 1998 to 2014 at a US academic center. Patients with chronic thromboembolic disease were excluded. When possible, a careful history and physical examination were performed to investigate for signs and symptoms consistent with chronic right ventricular insufficiency (history of PE, peripheral edema, ascites). Careful attention also was paid to radiographic markers of chronic disease (enlarged main pulmonary artery, paucity of lung vascularity). Unadjusted outcomes were described, and quantitative comparisons were made of short- and long-term echocardiography data. This study was approved by the Emory University Institutional Review Board under expedited review.

\section{Surgical Intervention}

Patients were evaluated for SPE as part of a multidisciplinary PE response team. Patients were considered for SPE when they had massive or submassive PEs with a large burden of thrombus, evidence of right ventricular dilation, and dysfunction on echocardiogram or computed tomography with a right ventricular to left ventricular ratio of 0.9 or greater and intrapericardial embolic disease within the pulmonary arterial tree. Although methods varied somewhat over the course of the study, all of the patients underwent SPE with the aid of cardiopulmonary bypass, mild to moderate systemic hypothermia, and blood cardioplegia when aortic crossclamping was used. Emboli were then removed from the main, right and left main, lobar, and segmental pulmonary arteries under direct vision using a combination of manual extraction of large thrombi and gentle suction evacuation techniques for small fragmented thrombi that were distal or too small to grasp with forceps. A counter incision in the right main pulmonary artery was used selectively. When the aorta was fully mobilized, visualization down to the sublobar level on the right side was usually clear, thus obviating the right main pulmonary artery incision in between the aorta and superior vena cava. Postoperative anticoagulation usually was initiated 6 hours after completion of SPE, and all patients had caval filters placed before discharge. Our decision to routinely insert inferior vena cava filters in these patients stems from registry data detailing improved in-hospital mortality rates for patients with pulmonary emboli who are unstable at presentation. ${ }^{15}$

\section{Echocardiographic Follow-up}

The institutional echocardiographic database was searched for perioperative and follow-up studies to compare markers of right ventricular function. These markers included qualitative assessments of right ventricular function and tricuspid regurgitation. Quantitative measures included estimations of pulmonary artery systolic pressure and velocity of the tricuspid regurgitant jet. Follow-up echocardiography was at the discretion of the treating internist or cardiologist.

\section{Statistical Analysis}

Continuous variables were summarized using mean \pm standard deviation. Differences between independent group means were evaluated using the 2-sample $t$ test; for paired group means (eg, preoperative and postoperative), the paired $t$ test was used. Categoric variables were summarized using count (percentage). Differences between independent group proportions were evaluated using the chi-square test; for paired group proportions, the McNemar test was conducted. When the number of events was small, the Fisher exact test and the exact McNemar test were performed for independent and correlated data, respectively. All tests of hypotheses were 2 sided and conducted at .05 level of significance. SAS 9.4 (SAS Institute Inc, Cary, NC) was used to perform all data analyses.

\section{RESULTS}

Forty-four patients met inclusion criteria and were submitted for analysis. Of those patients, $9(20.5 \%)$ had a massive PE requiring preoperative inotropy. Table 1 shows 
TABLE 1. Demographic data

\begin{tabular}{lccc}
\hline & All $(\mathbf{n}=\mathbf{4 4})$ & Massive $(\mathbf{n}=\mathbf{9})$ & Submassive (n=35) \\
\hline Age, y & $51.6 \pm 16.1$ & $50.6 \pm 14.8$ & $51.2 \pm 16.6$ \\
Female & $25(56.8 \%)$ & $6(66.7 \%)$ & $19(54.3 \%)$ \\
Status & & & .92 \\
$\quad$ Urgent & $16(36.4 \%)$ & $3(33.3 \%)$ & $13(37.1 \%)$ \\
$\quad$ Emergency & $28(63.6 \%)$ & $6(66.7 \%)$ & $22(62.8 \%)$ \\
Ejection fraction & $59.6 \pm 6.7$ & $60.0 \pm 11.5$ & $59.5 \pm 5.2$ \\
Diabetes & $8(18.2 \%)$ & 0 & $8(22.9 \%)$ \\
Chronic lung disease & & & .86 \\
$\quad$ None & $36(81.8 \%)$ & $6(66.7 \%)$ & $30(85.7 \%)$ \\
$\quad$ Mild & $6(13.6 \%)$ & $3(33.3 \%)$ & $3(8.6 \%)$ \\
$\quad$ Moderate & 0 & 0 & 0 \\
$\quad$ Severe & $2(4.6 \%)$ & 0 & $2(5.7 \%)$ \\
Previous myocardial infarction & $10(22.7 \%)$ & $2(22.2 \%)$ & $8(22.9 \%)$ \\
Previous CVA & $4(9.1 \%)$ & $1(11.1 \%)$ & $3(8.6 \%)$ \\
Previous cardiac surgery & $1(2.3 \%)$ & $0(0.0 \%)$ & $1(2.9 \%)$ \\
Cerebrovascular disease & $4(9.1 \%)$ & $1(11.1 \%)$ & $3(8.6 \%)$ \\
Mean preoperative serum creatinine & $1.1 \pm 0.6$ & $0.9 \pm 0.3$ & $1.2 \pm 0.7$ \\
History of smoking & $4(9.1 \%)$ & $4(44.4 \%)$ & 0 \\
Immunosuppressive therapy & $5(11.4 \%)$ & $0(0.0 \%)$ & .13 \\
Body mass index & $30.5 \pm 11.3$ & $29.8 \pm 5.8$ & .93 \\
\hline
\end{tabular}

CVA, Cerebrovascular accident.

the baseline demographics of the entire cohort and of the massive and submassive subgroups. Of note, the patients who underwent SPE were relatively young (aged 51 years). Of note, no patient in this study had preoperative cardiopulmonary arrest.

All patients underwent SPE with the aid of cardiopulmonary bypass, and 14 procedures $(31.8 \%)$ were performed with cardioplegic arrest. Mean cardiopulmonary bypass time was 68 minutes for the entire cohort because few patients $(5[11.4 \%])$ had concomitant cardiac surgical procedures performed. Further operative details are outlined in Table 2.

In-hospital outcomes are detailed in Table 3. There was a single 30 -day mortality $(2.3 \%)$ for the entire cohort. That patient had a combined coronary artery bypass grafting/ SPE and died of multisystem organ failure 2 weeks after the initial procedure. The rates of other in-hospital morbidities were low, including no incidence of postoperative stroke in the entire cohort.

Echocardiographic results are detailed in Tables 4 and 5. In general, patients who underwent SPE showed immediate improvement in right ventricular hemodynamics, including improved right ventricular function, decreased tricuspid regurgitation, and subsequent lowering of pulmonary artery pressures. Midterm echocardiographic follow-up was complete in 12 patients $(27.3 \%)$, and mean echocardiographic follow-up was 30.3 months. Table 5 compares the preoperative echocardiographic data with the midterm follow-up and shows a persistent improvement in right ventricular hemodynamics. Table 6 demonstrates the patient-specific echocardiographic follow-up data.

\section{DISCUSSION}

$\mathrm{PE}$ is a well-documented, well-studied disease with varying treatment algorithms based on treating specialty, available expertise, and extent of disease. Several centers recently have initiated multidisciplinary pulmonary embolus response teams (PERTs) to potentially broaden the treatment options for patients with life-threatening PEs. ${ }^{16}$ Surgeons are playing an ever-increasing role in PERT teams and in managing massive and submassive PEs.

In addition to mechanical embolectomy, both systemic and local lytic agents have been used to treat submassive and massive pulmonary emboli. The Pulmonary Embolism

TABLE 2. Operative data

\begin{tabular}{|c|c|c|c|c|}
\hline & All $(n=44)$ & Massive $(\mathbf{n}=9)$ & Submassive $(\mathbf{n}=\mathbf{3 5})$ & $P$ value \\
\hline Aortic crossclamp (min) & $47.4 \pm 40.4$ & $59.0 \pm 41.3$ & $43.9 \pm 40.7$ & .48 \\
\hline Cardiopulmonary bypass time (min) & $68.0 \pm 40.2$ & $82.8 \pm 54.9$ & $64.1 \pm 35.4$ & .22 \\
\hline Concomitant valve procedures & $3(6.8 \%)$ & $1(11.1 \%)$ & $2(5.7 \%)$ & .45 \\
\hline Concomitant CABG & $2(4.5 \%)$ & $1(11.1 \%)$ & $1(2.9 \%)$ & .27 \\
\hline Intraoperative IABP insertion & $5(11.4 \%)$ & $1(11.1 \%)$ & $4(11.4 \%)$ & .84 \\
\hline
\end{tabular}

$C A B G$, Coronary artery bypass grafting; $I A B P$, intra-aortic balloon pump. 
TABLE 3. Postoperative outcomes

\begin{tabular}{lcccc}
\hline & All $(\mathbf{n}=\mathbf{4 4})$ & $\begin{array}{c}\text { Massive } \\
(\mathbf{n}=\mathbf{9})\end{array}$ & $\begin{array}{c}\text { Submassive } \\
(\mathbf{n}=\mathbf{3 5})\end{array}$ & $\boldsymbol{P}$ value \\
\hline 30-d mortality & $1(2.3 \%)$ & 0 & $1(2.9 \%)$ & .91 \\
Stroke & 0 & 0 & 0 & \\
Renal failure & $4(9.1)$ & 0 & $4(11.4 \%)$ & .54 \\
New dialysis & $1(2.3 \%)$ & 0 & $1(2.9 \%)$ & .91 \\
Prolonged ventilation & $17(38.6 \%)$ & $3(33.3 \%)$ & $14(40.0 \%)$ & .77 \\
Reexploration for & $2(4.5)$ & 0 & $2(5.7 \%)$ & .83 \\
$\quad$ hemorrhage & \multicolumn{4}{c}{} \\
\hline
\end{tabular}

Thrombolysis trial was a randomized prospective trial that compared the use of systemic tenecteplase with systemic heparin. ${ }^{17}$ Although systemic lytic agents prevented hemodynamic collapse from PE, there was an $11.5 \%$ risk of major hemorrhage, a $2.4 \%$ risk of stroke, and a $2.4 \%$ all-cause mortality in the lysis arm of the trial. Data regarding catheter-directed thrombolysis in the treatment of submassive and massive PE have been limited to single-center case series with small numbers of patients. A 2009 metaanalysis with 594 patients showed that catheter-directed lysis was safe and had a lower complication rate when compared with historical data for systemic lysis. ${ }^{18}$

Researchers have documented single-center outcomes after SPE. ${ }^{14,19-22}$ These reports document excellent outcomes with low mortality rates in extremely ill patient populations. Despite these reports, publications citing disparate outcomes persist. In a recent analysis of the Nationwide Inpatient Sample, researchers at Johns Hopkins showed an inpatient mortality rate of $27.2 \%$, which is more than double the mortality quoted in most of the single-center series. ${ }^{23}$ In the conclusion of that article, Kilic and colleagues ${ }^{23}$ recommended against transfer of these patients to centers of excellence when qualified surgical therapy was available because of the lack of impact of surgeon or hospital volume on mortality in their multivariate model. Also, although it is not stated explicitly in the medical literature, the opinion exists among some treating specialists that SPE is not only dangerous but also ineffective. Many continue to voice concerns over the long-term development of chronic thromboembolic pulmonary

TABLE 4. Postoperative echocardiographic data

\begin{tabular}{|c|c|c|c|}
\hline & $\begin{array}{l}\text { Preoperative } \\
\quad(\mathbf{n}=\mathbf{2 1})\end{array}$ & $\begin{array}{l}\text { Postoperative } \\
(\mathbf{n}=\mathbf{2 1})\end{array}$ & $P$ value \\
\hline $\begin{array}{l}\text { Moderate or greater } \\
\text { ventricular dysfunction }\end{array}$ & $17(81.0 \%)$ & $9(42.9 \%)$ & .01 \\
\hline $\begin{array}{l}\text { Moderate or greater tricuspid } \\
\text { regurgitation }\end{array}$ & $6(28.6 \%)$ & $1(4.8 \%)$ & .03 \\
\hline $\begin{array}{l}\text { Pulmonary artery systolic } \\
\text { pressure }(\mathrm{mm} \mathrm{Hg})\end{array}$ & $\begin{array}{l}51.2 \pm 21.6 \\
(\mathrm{n}=17)\end{array}$ & $\begin{aligned} 36.6 & \pm 10.1 \\
(\mathrm{n} & =6)\end{aligned}$ & .21 \\
\hline $\begin{array}{l}\text { Tricuspid valve regurgitant } \\
\text { velocity }(\mathrm{m} / \mathrm{s})\end{array}$ & $\begin{array}{l}3.1 \pm 0.8 \\
(\mathrm{n}=16)\end{array}$ & $\begin{aligned} 2.8 & \pm 0.7 \\
(\mathrm{n} & =7)\end{aligned}$ & .20 \\
\hline
\end{tabular}

TABLE 5. Midterm echocardiographic follow-up

\begin{tabular}{lccc}
\hline & $\begin{array}{c}\text { Preoperative } \\
\text { value }(\mathbf{n}=\mathbf{2 1})\end{array}$ & $\begin{array}{c}\text { Midterm } \\
\text { value }(\mathbf{n}=\mathbf{1 2})\end{array}$ & $\boldsymbol{P}$ value \\
\hline $\begin{array}{l}\text { Moderate or greater } \\
\text { ventricular dysfunction }\end{array}$ & $17(81.0 \%)$ & $1(8.3 \%)$ & $<.0001$ \\
Moderate or greater tricuspid & $6(28.6 \%)$ & $0(0)$ & .0005 \\
$\quad$ regurgitation & & & \\
Pulmonary artery systolic & $51.2 \pm 21.6$ & $37.2 \pm 14.2$ & .01 \\
$\quad$ pressure (mm Hg) & $(\mathrm{n}=17)$ & $(\mathrm{n}=9)$ & \\
Tricuspid valve regurgitant & $\begin{array}{c}3.1 \pm 0.8 \\
(\mathrm{n}=16)\end{array}$ & $\begin{array}{c}2.4 \pm 0.7 \\
(\mathrm{n}=11)\end{array}$ & .03 \\
$\quad$ velocity $(\mathrm{m} / \mathrm{s})$ & & & \\
\hline
\end{tabular}

hypertension even after SPE. Little data exist regarding midterm echocardiographic follow-up after SPE, but there is evidence to suggest that patients with submassive PEs and significant right ventricular dysfunction on admission (systolic pulmonary artery pressure $>50 \mathrm{~mm} \mathrm{Hg}$ ) develop right ventricular dysfunction or pulmonary hypertension approximately $50 \%$ of the time at 1-year follow-up. ${ }^{24}$

This article and research sought to directly refute the described notions by showing that SPE is a safe procedure with low morbidity and mortality. In addition, this study illustrated both the short-term and midterm echocardiographic follow-up for patients who underwent SPE to demonstrate a durable benefit in right ventricular function. These data demonstrated that SPE for acute PE was extremely safe over the course of the study with 1 inhospital mortality and few other perioperative morbidities.

The echocardiographic data in this study demonstrated the hemodynamic effectiveness of the procedure. In the short term, the effects of SPE on right ventricular function are dramatic. Qualitatively, right ventricular function improved and tricuspid regurgitation decreased. There were also significant quantitative declines in pulmonary artery pressure and tricuspid regurgitant velocity. On midterm follow-up echocardiography, these improvements in right ventricular hemodynamics persisted, thus proving that the immediate effects of SPE are durable across a longer time period.

\section{Study Limitations}

Given the retrospective nature of this study, there were some limitations. There were a number of surgeons involved in the treatment of the patients over a long study period, thus introducing the possibility of variations in surgical technique and the potential for selection bias in who was offered surgical therapy. Certain surgeons could have offered or declined surgical intervention on the basis of variables unable to be captured by this or other databases. There was no standardized echocardiographic follow-up, nor was there a centralized echocardiography core for review of films. As such, there could have been variation in the interpretation of qualitative analyses of right ventricular 
TABLE 6. Patient-specific echocardiographic follow-up data

\begin{tabular}{|c|c|c|c|c|c|}
\hline Patient & $\begin{array}{c}\text { Months at last } \\
\text { echocardiography }\end{array}$ & $\begin{array}{l}\text { Preoperative PA } \\
\text { systolic pressure }\end{array}$ & $\begin{array}{c}\text { Midterm PA } \\
\text { systolic pressure }\end{array}$ & Preoperative TV velocity & Midterm TV velocity \\
\hline 1 & 11 & 110 & & 5 & 3.2 \\
\hline 2 & 100 & & 26.6 & & 2.6 \\
\hline 3 & 93 & 60 & 45 & 3.5 & \\
\hline 4 & 73 & 89 & 62.6 & 4.3 & 3.4 \\
\hline 5 & 53 & & 41.2 & & 2.3 \\
\hline 6 & 1 & 46.1 & 42.5 & 2.4 & 2.9 \\
\hline 7 & 18 & 35 & 12.7 & & 0.8 \\
\hline 8 & 2 & 25.7 & 28.5 & 2 & 2.1 \\
\hline 9 & 3 & 57.6 & 43.6 & 3.5 & 2.9 \\
\hline 10 & 4 & 44.4 & & 2.7 & 1.8 \\
\hline 11 & 2 & 59 & 31.8 & 3.5 & 2.4 \\
\hline 12 & 3 & 33.7 & & 2.8 & 1.9 \\
\hline
\end{tabular}

$P A$, Pulmonary artery; $T V$, tricuspid valve.

function between reviewers. Given the variations in treatment over time, the long-term medical management of patients after SPE was not standardized. There were no uniform approaches to duration of postoperative anticoagulation in this patient population.

On the basis of this study and our growing experience in the multispecialty management of patients with acute PE, we have changed our practice. We are more likely to incorporate SPE into the treatment algorithm for patients with massive and submassive PE. Also, we have standardized the echocardiographic follow-up around the formation of a PE clinic. All patients with a PE seen by our institutional PERT are now seen in a specialized clinic with members of the PERT team and receive directed therapy with 6-month echocardiographic follow-up at a minimum. Since mid2012, which coincided with the commencement of our PERT, PERT activations have been approximately 150 per year. Since that time, our center performs between 4 and 6 SPEs annually in addition to 25 to 30 catheter-based interventions per year.

Patients at our institution currently are considered for SPE according to a few factors. The majority of patients have high-risk submassive PEs as defined by right ventricular strain or dilation (a right ventricular to left ventricular ratio of $\geq 0.9$ per computed tomography or right ventricular dysfunction on echocardiogram), evidence of serologic cardiac damage, and some degree of cardiopulmonary compromise including tachycardia and hypoxia. On the basis of data from researchers at Vanderbilt, we generally consider operative intervention in patients with emboli in the intrapericardial segments of the pulmonary artery. ${ }^{12}$ In keeping with the guidelines for valve disease issued by the American College of Cardiology and the American Heart Association, patients who undergo SPE derive the most benefit from operative therapy given an estimated survival of greater than 1 year when this can be determined. ${ }^{25}$ Common contraindications to SPE include but are not limited to intracranial hemorrhage, multisystem organ dysfunction, and severe preexisting pulmonary disease. We are also careful in the preoperative evaluation of patients to avoid those with chronic thromboembolic disease. Specifically, we try to avoid performing emergency operation in patients with significant peripheral edema, a history of pulmonary emboli, ascites, a significantly enlarged main pulmonary artery, and a chronically avascular lung fields. Through this combination of history, physical examination, and radiographic findings, we have been largely able to exclude patients with chronic disease from undergoing emergency operations. We also recommend the availability of extracorporeal membrane oxygenation as an adjunct to assist in recovery from severe right heart dysfunction after SPE.

\section{CONCLUSIONS}

SPE is an effective procedure in alleviating the adverse hemodynamic effects on right ventricular function after a large massive or submassive PE. It is safe with low rates of perioperative morbidity and in-hospital mortality. The beneficial effects of SPE on right ventricular hemodynamics persist well beyond the perioperative period. On the basis of these data, we recommend that SPE be considered for patients with appropriate anatomy and submassive and massive PEs.

\section{Webcast}

You can watch a Webcast of this AATS meeting presentation by going to: http://webcast.aats.org/2015/Video/Tuesday/ 04-28-15_612_1642_Keeling.mp4.

\section{Conflict of Interest Statement}

Authors have nothing to disclose with regard to commercial support.

\section{References}

1. Rubinstein I, Murray D, Hoffstein V. Fatal pulmonary emboli in hospitalized patients. An autopsy study. Arch Intern Med. 1988;148:1425-6.

2. Dalen JE, Alpert JS. Natural history of pulmonary embolism. Prog Cardiovasc Dis. 1975;17:259-70. 
3. Bell WR, Simon TL. Current status of pulmonary thromboembolic disease: pathophysiology, diagnosis, prevention, and treatment. Am Heart J. 1982;103:239-62.

4. Dimick JB, Chen SL, Taheri PA, Henderson WG, Khuri SF, Campbell DA Jr. Hospital costs associated with surgical complications: a report from the private-sector National Surgical Quality Improvement Program. J Am Coll Surg. 2004;199:531-7.

5. Anderson FA Jr, Wheeler HB, Goldberg RJ, Hosmer DW, Patwardhan NA, Jovanovic B, et al. A population-based perspective of the hospital incidence and case-fatality rates of deep vein thrombosis and pulmonary embolism. The Worcester DVT Study. Arch Intern Med. 1991;151:933-8.

6. Goldhaber SZ, Visani L, De Rosa M. Acute pulmonary embolism: clinical outcomes in the International Cooperative Pulmonary Embolism Registry (ICOPER). Lancet. 1999;353:1386-9.

7. Kucher N, Rossi E, De Rosa M, Goldhaber SZ. Massive pulmonary embolism. Circulation. 2006;113:577-82.

8. Stein PD, Matta F, Janjua M, Yaekoub AY, Jaweesh F, Alrifai A. Outcome in stable patients with acute pulmonary embolism who had right ventricular enlargement and/or elevated levels of troponin I. Am J Cardiol. 2010;106:558-63.

9. Grifoni S, Vanni S, Magazzini S, Olivotto I, Conti A, Zanobetti M, et al. Association of persistent right ventricular dysfunction at hospital discharge after acute pulmonary embolism with recurrent thromboembolic events. Arch Intern Med. 2006;166:2151-6.

10. van Dongen CJ, van den Belt AG, Prins MH, Lensing AW. Fixed dose subcutaneous low molecular weight heparins versus adjusted dose unfractionated heparin for venous thromboembolism. Cochrane Database Syst Rev. 2004;CD001100.

11. Sharp EH. Pulmonary embolectomy: successful removal of a massive pulmonary embolus with the support of cardiopulmonary bypass. Case report. Ann Surg. $1962 ; 156: 1-4$.

12. Gibbon JH Jr. Extracorporeal maintenance of cardiorespiratory functions. Harvey Lect. 1957;53:186-224.

13. Aklog L, Williams CS, Byrne JG, Goldhaber SZ. Acute pulmonary embolectomy: a contemporary approach. Circulation. 2002;105:1416-9.

14. Greelish JP, Leacche M, Solenkova NS, Ahmad RM, Byrne JG. Improved midterm outcomes for type A (central) pulmonary emboli treated surgically. $J$ Thorac Cardiovasc Surg. 2011;142:1423-9.

15. Stein PD, Matta F. Thrombolytic therapy in unstable patients with acute pulmonary embolism: saves lives but underused. Am J Med. 2012;125:465-70.

16. Provias T, Dudzinski DM, Jaff MR, Rosenfield K, Channick R, Baker J, et al. The Massachusetts General Hospital Pulmonary Embolism Response Team (MGH PERT): creation of a multidisciplinary program to improve care of patients with massive and submassive pulmonary embolism. Hosp Pract (1995). 2014; 42:31-7.

17. Meyer G, Vicaut E, Konstantinides SV. Fibrinolysis for intermediate-risk pulmonary embolism. $N$ Engl J Med. 2014;371:581-2.

18. Kuo WT, Gould MK, Louie JD, Rosenberg JK, Sze DY, Hofmann LV. Catheterdirected therapy for the treatment of massive pulmonary embolism: systematic review and meta-analysis of modern techniques. J Vasc Interv Radiol. 2009; 20:1431-40.

19. Aklog L. Emergency surgical pulmonary embolectomy. Semin Vasc Med. 2001; $1: 235-46$.

20. Osborne ZJ, Rossi P, Aucar J, Dharamsy S, Cook S, Wheatley B. Surgical pulmonary embolectomy in a community hospital. Am J Surg. 2014; 207:337-41.

21. Takahashi H, Okada K, Matsumori M, Kano H, Kitagawa A, Okita Y. Aggressive surgical treatment of acute pulmonary embolism with circulatory collapse. Ann Thorac Surg. 2012;94:785-91.

22. Zarrabi K, Zolghadrasli A, Ali Ostovan M, Azimifar A, Malekmakan L. Residual pulmonary hypertension after retrograde pulmonary embolectomy: long-term follow-up of 30 patients with massive and submassive pulmonary embolism. Interact Cardiovasc Thorac Surg. 2013;17:242-6.

23. Kilic A, Shah AS, Conte JV, Yuh DD. Nationwide outcomes of surgical embolectomy for acute pulmonary embolism. J Thorac Cardiovasc Surg. 2013;145:373-7.

24. Ribeiro A, Lindmarker P, Johnsson H, Juhlin-Dannfelt A, Jorfeldt L. Pulmonary embolism: one-year follow-up with echocardiography Doppler and five-year survival analysis. Circulation. 1999;99:1325-30.

25. Nishimura RA, Otto CM, Bonow RO, Carabello BA, Erwin JP III, Guyton RA, et al. 2014 AHA/ACC guideline for the management of patients with valvular heart disease: a report of the American College of Cardiology/American Heart Association Task Force on Practice Guidelines. J Am Coll Cardiol. 2014;63: e57-185.

\section{Discussion}

Dr Rune Haaverstad (Bergen, Norway). SPE has gained renewed interest after going through different phases. Professor Trendelenburg of Leipzig invented a dangerous procedure more than 100 years ago with high mortality.

Pulmonary embolism motivated the invention of the heart-lung machine with successful cases done since the 1960s. Later, surgical embolectomy became near extinction because of the use of fibrinolysis, with renewed interest in recent years with several good clinical series if performed before the need of cardiopulmonary resuscitation.

This well-presented work of yours is adding to the recent success. The purpose of your study was to document postoperative midterm and long-term effects on the right heart function, and because it has been a concern that the right heart also could suffer after surgery.

However, the strength of the results are maybe not very high because less than $50 \%$ had postoperative echocardiograms, and approximately $27 \%$ had a midterm echocardiogram at 30 months. Nevertheless, the results are consistent, and the right heart normalized in general, and it was a good result both clinically and hemodynamically. So to my questions, they are regarding inclusion, surgery, and assessment.

New York Heart Association class III and IV was seen in only $55 \%$ of the massive cases and $37 \%$ of the submassive cases. Because pulmonary embolism is frequent and symptoms are varying, did you follow any treatment algorithm in this period of time for pulmonary embolism or was inclusion for surgical embolectomy by coincidence?

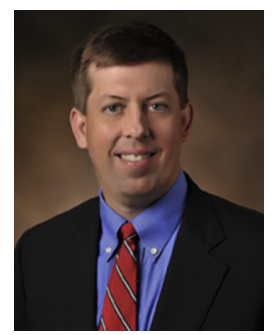

Dr Keeling. In terms of the percentage of patients who preoperatively had New York Heart Association class III and IV failure, I don't know how that was coded. The time course of this study occurred over a long period of time ( $\sim 16$ years), and I'm not sure that all of those patients had the extent of heart failure coded. Some of the coding may be mistaken in terms of right ventricular dysfunction, so I can't speak to that per se.

Dr Haaverstad. I received your article in advance, so I do have more information on that. It's also been noted that because I had to look at the surgery, all thrombus was removed. You don't see too much through the proximal pulmonary circulation. How well has the effect of the surgery been assessed intraoperatively and postoperatively, for instance by computed tomography scanning?

Dr Keeling. In our current protocol, we just use cardiopulmonary bypass. We don't in general arrest the heart with cardioplegia and tend to make a single-pump pulmonary arteriotomy through the main pulmonary artery.

We fully mobilize the aorta. The left pulmonary artery rarely is problematic, and I think that you can get down to 
the segmental arteries on the left side rather easily. It's the right pulmonary artery that tends to be difficult in terms of extraction of all the embolus on that side.

I know that Trendelenburg described a counter-incision on the right main pulmonary artery. We make that selectively, and I can't say how frequently we do it, probably approximately $25 \%$ of the time. But we tend to be able to completely visualize the right side with full mobilization of the aorta, and when we put an aortic root retractor in the pulmonary arteriotomy, we are able to see, again, down to the segmental level on the right side.

We do use gentle suction extraction of clots. We're very diligent about that and do it only under direct vision, knowing that there have been some reports in terms of intimal injury with suction devices. But we do it selectively and gently, and have been rather successful with it.

Dr Haaverstad. Furthermore, the cardioplegic arrest was done in $32 \%$ of all the cases. Because avoidance of aortic crossclamping is stressed by some surgeons, did you expect or find any difference in outcome between those having cardioplegic arrest or not?

Dr Keeling. Our techniques have evolved to the point that we rarely have to use that, only in extreme cases when we're having difficulty with visualization. The design of this study was not to evaluate those patients who had their hearts arrested versus those who didn't, and so we haven't analyzed those data in that fashion. But you bring up an excellent point. It's something we should look into.

Dr Haaverstad. In view of the extracorporeal membrane oxygenation indications, which hospitals should do this type of surgery?
Dr Keeling. I think it was the point of the Johns Hopkins researchers in 2012, and in their conclusion they say you shouldn't transfer these patients given that the mortality is high, and it doesn't really matter where these patients go so long as there are qualified surgeons present.

I do think that advanced cardiopulmonary support measures are indicated for some of these patients, and certainly for high-risk submassive pulmonary emboli, you should try and transfer these patients when possible. For those with massive pulmonary emboli, you probably just don't have the opportunity to do so, and systemic fibrinolysis likely is the appropriate treatment for those patients. But if you're forced to operate on them, they should be in a capable surgeon's hands at whatever center is closest.

Dr Eric Sievers (Jackson, Tenn). Quick question on the timing from diagnosis to surgery to touch on the last point. In your submassive group, did any of those have thrombolysis before surgery?

Dr Keeling. Not to my knowledge. In terms of from the point of diagnosis to the point of intervention, in those patients with massive pulmonary emboli, you don't have a lot of choice. You've got to do something. You have to do it rather quickly. For patients with submassive PE, we usually try to operate on them during the light of day.

Many times, this is an urgent procedure. It's not elective or urgent. So if we find out about the patient in the morning, we'll do it in the afternoon. If we find out about the patient in the middle of the night, we'll do it the next morning. I think we've been successful with those algorithms in terms of timing of treatment. 